\section{Comparison of 64-Slice CT with Gated SPECT for Evaluation of Left Ventricular Function}

TO THE EDITOR: We were interested in the conclusions of Schepis et al. (1), who stated that there is good agreement between left ventricular (LV) ejection fraction (LVEF) and LV functional parameters estimated using CT and gated SPECT across a wide range of clinically relevant values. They appear to base this statement on the good correlation between parameters estimated using the 2 techniques. However, good correlation does not, in itself, indicate good clinical agreement (2).

The authors presented Bland-Altman plots and reported limits of agreement. These are a good indicator of the level of clinical agreement, but the results shown were not adequately discussed. We would argue that limits of agreement of $\pm 15.1 \%$ on LVEF, where the threshold of normality is $50 \%$ and mean values in the study were 59\% (SPECT) and 60\% (CT), do not indicate good agreement, because the potential level of difference in individual cases is large. Similarly, the limits of agreement for end-diastolic volume and end-systolic volume of $\pm 51.7 \mathrm{~mL}$ and $\pm 32 \mathrm{~mL}$, respectively, do not indicate good agreement.

The authors' conclusions are somewhat inconsistent. They suggest that although the techniques agree for LVEF, they should not be used interchangeably for LV volumes; LVEF is calculated from LV volumes.

Intraobserver reproducibility was reported as excellent for SPECT. The SD of $4.6 \%$ is again high relative to the normal threshold; a potential error of greater than $4.6 \%$ in 1 in 3 patients is significant. Interobserver error was not reported for SPECT but is likely to be higher than intraobserver error. The $6.4 \% \mathrm{SD}$ of interobserver differences for CT is high. The authors should investigate the sources of these differences; in our experience, intra- and interobserver differences of this magnitude are unusual.

A further point of interest is the systematic difference between the 2 techniques in the estimation of muscle mass. In determining likely explanations, it would be useful to know the extent of myocardium included and whether analysis of the SPECT images included nonperfused muscle.

We would also like to point out an error in the presentation of data. The percentage mean differences shown in Table 2 are given in the text as SD on the absolute mean difference; the actual SDs are considerably larger. This may lead to an incorrect conclusion regarding intraobserver reproducibility.

In conclusion, we believe that the data show poor intraobserver reproducibility in the estimation of LVEF and very poor clinical agreement between SPECT and CT for the estimation of LVEF and LV functional parameters. LVEF and LV functional parameters as determined by 64-slice CT do not agree with gated SPECT and should not be used interchangeably. Furthermore, the large random differences between the techniques suggest that neither provides a clinically reliable measure of LVEF in this study.

\section{REFERENCES}

1. Schepis T, Gaemperli O, Koepfli P, et al. Comparison of 64-slice CT with gated SPECT for evaluation of left ventricular function. J Nucl Med. 2006;47:1288-1294.

2. Bland JM, Altman DG. Statistical methods for assessing agreement between two methods of clinical measurement. Lancet. 1986;1:307-310.

DOI: 10.2967/jnumed.106.037333

Nick Dudley Charnie Kalirai

Nottingham University Hospitals Nottingham, United Kingdom

REPLY: We appreciate the important comments regarding our recent comparison of left ventricular function assessment by 64 slice CT versus gated SPECT (1). We agree with Dudley and Kalirai that good correlation does not necessarily indicate good agreement of 2 measurements. Therefore, we have reported Bland and Altman limits of agreement in our study. The latter, however, provide no objective cutoff value above which intermethod agreement is considered significant or insufficient. Therefore, it must remain a matter of clinical judgment to evaluate in each clinical setting whether given limits of agreement are acceptable. In this specific comparison, we believe that the difference may be less relevant at least for values of left ventricular ejection fraction in the higher range than for values in the lower normal range.

Regarding the question of whether nonperfused muscle was included, we should clarify that a transmural scar cannot be depicted by SPECT. We have discussed the fact that one possible explanation for apparent overestimation of left ventricular volumes using CT is that delineation of the myocardial contours by SPECT may be hampered by the presence of severe perfusion defects. This may apply equally to the muscle mass measurement.

In summary, we agree that the statement in our conclusion, namely that the 2 techniques should not be used interchangeably because of variances inherent in the different techniques, should be emphasized and that this statement might possibly be extended, with caution, to left ventricular ejection fraction.

We apologize that the percentage mean difference shown in Table $2(+1.7 \%)$ is erroneously given in the text as SD on the absolute mean difference, which should read $1.1 \% \pm 7.7 \%$. Finally, an erratum has been brought to our attention: The myocardial mass calculated by CT was significantly lower (not higher), compared with gated SPECT $(127 \pm 24 \mathrm{~g}$ vs. $148 \pm 37 \mathrm{~g}$; mean difference, $23.0 \pm 12.2 \mathrm{~g} ; P<0.01$ ), as correctly stated in Table 2 .

\section{REFERENCE}

1. Schepis T, Gaemperli O, Koepfli P, et al. Comparison of 64-slice CT with gated SPECT for evaluation of left ventricular function. J Nucl Med. 2006;47:12881294.

Philipp A. Kaufmann Oliver Gaemperli University Hospital Zurich Zurich, Switzerland 